\title{
Respiratory and cardiac abnormalities in brain-stem ischaemia
}

\author{
AMOS D. KORCZYN ${ }^{1}$ \\ From the Department of Neurology, Beilinson Hospital, Petah Tikva, and \\ Department of Physiology and Pharmacology, Tel Aviv University Medical School, Israel
}

SYNOPSIS Two patients are presented who had suffered episodes of ischaemia of the brain-stem, mainly affecting one side of the pons. In addition to the more usual neurological signs, these two patients had cardiac arrhythmia: one had a sinus arrhythmia, the other a wandering pacemaker. In both patients the hemidiaphragm on the side ipsilateral to the lesion was transiently elevated. The mechanisms responsible for the arrhythmia and the elevated hemidiaphragm are discussed.

In previous studies, lesions of a cerebral hemisphere were shown to affect the respiratory activity of the contralateral hemidiaphragm (Korczyn et al., 1969a, b). It was concluded that a decussation of the corticofugal fibres takes place, similar to the pyramidal decussation. However, the exact level at which these pathways cross the midline could not be ascertained in these studies. Since then we have had the opportunity of observing two cases with vascular lesions affecting the pons which have added to our understanding of the suprasegmental control of respiration. In addition, both patients demonstrated cardiac arrhythmias, possibly resulting from the brain-stem lesion.

\section{CASE 1}

A 60 year old woman was admitted to hospital with severe vertigo of acute onset, accompanied by nausea and vomiting. Her previous history is unremarkable except for a milder vertiginous episode three years before.

On examination she was obese, with blood pressure readings of $140 / 80-190 / 100 \mathrm{mmHg}$ and a completely irregular heart rate. Neurological findings included nystagmus to the right in the primary position, which was enhanced in the right lateral gaze, with counterclockwise rotatory component.

1 Address for reprints: A. D. Korczyn, Department of Physiology and Pharmacology, Sackler School of Medicine, Tel Aviv University, Israel.

(Accepted 15 August 1974.)
There was hypoaesthesia on the right side of the face and of the pharynx, and slight weakness and increased tendon jerks on the left, with extensor plantar response on that side.

Blood and urine tests were non-contributory, except for a transient hyperglycaemia (fasting plasma glucose $145 \mathrm{mg} / \mathrm{dl}$, which gradually fell to $106 \mathrm{mg} / \mathrm{dl})$.

Chest radiographs revealed elevation of the right hemidiaphragm (Fig. 1). The heart was enlarged. On fluoroscopy the right hemidiaphragm failed to move. The patient volunteered the information that with the vertigo she noted irregular heart beats for the first time in her life. Electrocardiography (ECG) demonstrated severe sinus arrhythmia (Fig. 2) but was otherwise normal. The arrhythmia was not related to the respiration. After the administration of $0.5 \mathrm{mg}$ atropine intravenously, tachycardia developed at a rate of $96 / \mathrm{min}$, with a regular rate. Thirty-six hours later the ECG again demonstrated the arrhythmia. Right brachial arteriography demonstrated the right carotid and vertebral arteries as well as the basilar artery and their intracranial branches.

The patient slowly improved and on examination two months later no neurological abnormalities were detected and the heart rate was regular. The right hemidiaphragm also regained some movement, although it was still higher than the left.

SUMMARY This patient probably had a lesion of the caudal brain-stem, particularly on the right. The hemidiaphragm on the right (contralateral to the affected limbs) was weak. The ECG showed marked sinus arrhythmia. 


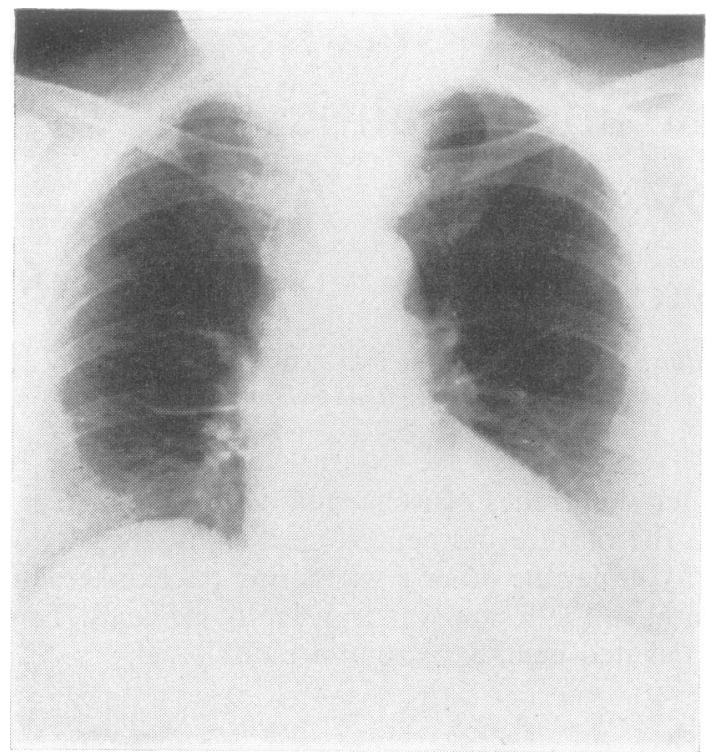

FIG. 1 (left) Case 1. Chest radiograph on inspiration on admission. Note elevation of right hemidiaphragm. The patient had weakness of the left limbs.

FIG. 2 (below) Case 1. Electrocardiogram on admission (lead 2). Note sinus arrhythmia. The lower trace is continuous with the upper one.

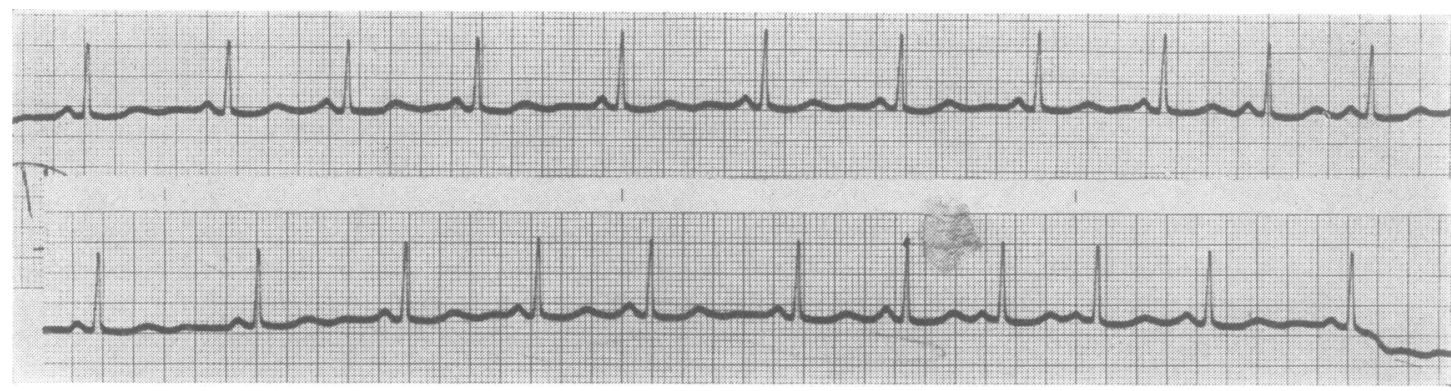

CASE 2

A 71 year old man was admitted to hospital after several episodes of left facial weakness and hypoaesthesia, vertigo, instability, dysarthria, and difficulty in swallowing over the preceding four months. His previous history was unremarkable.

On examination he was drowsy, with normal blood pressure and irregular heart rate. There was horizontal nystagmus on lateral gaze to either direction. Left facial weakness of lower motor neurone type was found, with hypoaesthesia over the face on the left and over the right side of the body. The right limbs were weak with brisker tendon jerks. An extensor plantar response was also evoked on the right. Ataxia of the right limbs was suspected but was difficult to evaluate in view of the weakness.

The chest radiograph showed that the left hemidiaphragm was elevated (Fig. 3). The ECG showed a sinus arrhythmia and multiple atrial premature beats (Fig. 4).

The patient improved slowly and was discharged from hospital five weeks later, with considerable persistent neurological disability. The ECG was unchanged, but the diaphragm was now normal (Fig. 5).

SUMMARY This patient showed evidence of vertebrobasilar ischaemia, mainly affecting the left side of the pons. There was weakness of the diaphragm on the side of the lesion (contralateral to the affected limbs) and his ECG showed sinus arrhythmia and multiple atrial premature beats.

\section{DISCUSSION}

We have shown previously that in patients with hemispheric lesions the contralateral hemi- 


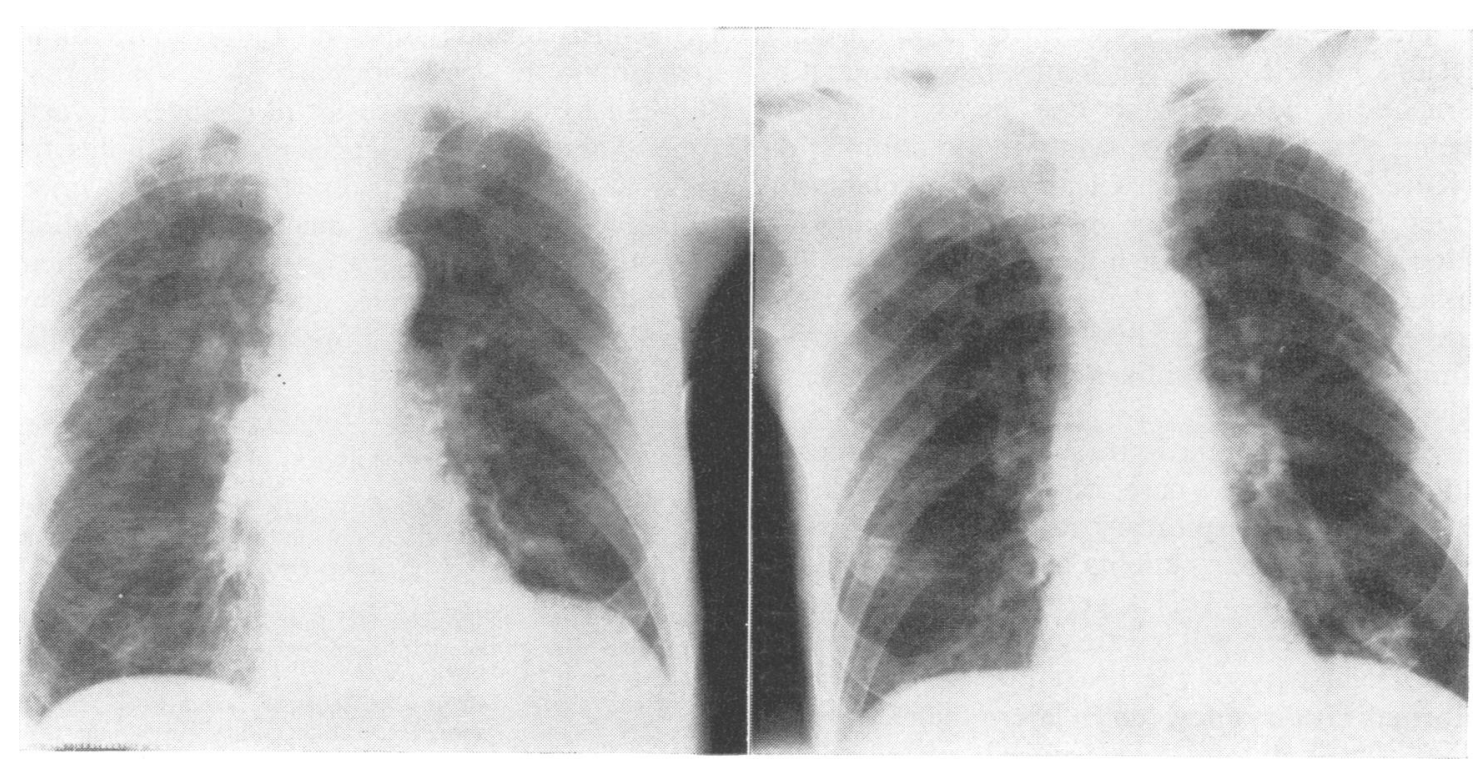

FIG. 3 (left) Case 2. Chest radiograph on inspiration on admission. Note elevation of left hemidiaphragm. The patient had right hemiparesis.

FIG. 5 (right) Case 2. Chest radiograph on inspiration five weeks after admission. Both hemidiaphragms are now at the same height.

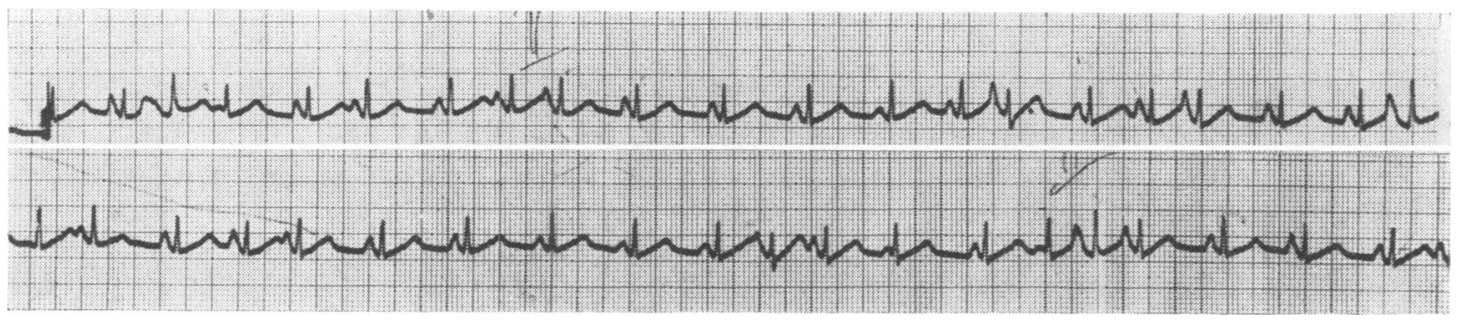

FIG. 4 Case 2. Electrocardiogram on admission (lead 2). Note the sinus arrhythmia with multiple atrial premature beats. There is possibly a nodal beat towards the end of the trace. The lower trace is continuous with the upper one.

diaphragm is frequently elevated and shows relatively less movement (Korczyn et al., 1969a, b). In the present patients the evidence suggests mainly unilateral brain-stem damage. Exact localization of the lesion could not be determined in either patient. In case 2 the evidence strongly suggests that it was located at the level of the facial nucleus, although there is the possibility that it may have been more extensive. The hemidiaphragm was elevated on the side ipsilateral to the lesion. This indicates that the descending corticofugal pathways affecting respiration cross the midline above the pyramidal decussation. This would not be surprising: the respiratory system does not consist of the diaphragm alone but includes several other muscles, the most cranial of which is the ala nasi which is innervated by the facial nerve, whose nucleus is in the pons. Thus it is likely that lesions such as those of our two patients will involve other muscles connected with respiration as well. Unfortunately, this was not looked for. 
The cardiac changes which occur after vascular lesions in the cerebral hemispheres and subarachnoid haemorrhage can be explained as being due to excessive sympathetic stimulation (Korczyn et al., 1971). Clearly, this explanation does not hold for the present cases, and an alternative mechanism must be looked for. The association between the brain lesions and the appearance of the arrhythmias, which occurred simultaneously, is unlikely to have been due to chance.

Sinus arrhythmia is frequently seen clinically in children. In this case, however, the rhythm is locked to the respiratory rate, in contrast with the finding in our patients in whom it was completely irregular. This, together with the fact that the first patient was aware of the appearance of the irregularity of the heart rate as well as the normalization a few days later, make it likely that the arrhythmia was indeed related to the ischaemic episode of the brain-stem. Sinus arrhythmia of the common type is generally thought to occur as a result of changes in vagal tone. The same explanation probably holds for this patient, since it disappeared after an injection of atropine. The mechanism by which arrhythmia was produced in the second patient is probably more complex and we prefer to defer speculations pending more reports of similar cases.

\section{REFERENCES}

Korczyn, A. D., Hermann, G., and Don, R. (1969a). Diaphragmatic involvement in hemiplegia and hemiparesis. Journal of Neurology, Neurosurgery, and Psychiatry, 32, 588-590.

Korczyn, A. D., Leibowitz, U., and Bruderman, I. (1969b). Involvement of the diaphragm in hemiplegia. Neurology (Minneap.), 19, 97-100.

Korczyn, A. D., Spitzer, S., Kott, E., and Bornstein, B. (1971). Electrocardiographic abnormalities in patients with brain-stem tumours. Confinia Neurologica, 33, 304-308. 